\title{
Controlador preditivo robustamente estável para processos com polos integradores repetidos *
}

\author{
Igor M. L. Pataro* Rafael S. de Lima* \\ Márcio A. F. Martins* \\ * PPGM - Programa de Pós-Graduação em Mecatrônica \\ Universidade Federal da Bahia \\ Salvador,Bahia,Brasil (e-mail: igor_pataro@yahoo.com.br, \\ rafaelsilvadelima91@gmail.com, marciomartins@ufba.br).
}

\begin{abstract}
This paper presents a robustly stabilizing model predictive control strategy for systems with multiple integrating poles. In this approach, the controller extends the results obtained by Costa et. al. (2018) to cases with model uncertainties, whose robustness is achieved through the cost-function contraction for all models beloging to the proposed multi-plant set. The work presents two formulations associated with the control optimization problem to guarantee the convergence of the closed-loop system. The approaches are applied to a Hover system as a case study scenario. It is shown that both approaches can guarantee the contraction of the cost function, thus leading to the convergence of the closed-loop system both for the reference tracking and unmeasured disturbances scenarios.

Resumo: Este trabalho apresenta uma estratégia de controle preditivo robustamente estável para sistemas com múltiplos polos integradores. Nesta abordagem, o controlador estende os resultados obtidos por Costa et. al. (2018) para casos com incertezas de modelo no qual a robustez é obtida pela contração da função custo para todos os modelos pertencentes ao conjunto multi-plantas. O trabalho realizado apresenta duas formulações associadas ao problema de otimização para garantir a convergência do sistema em malha fechada. As abordagens são aplicadas em um sistema Hover como cenário de estudo de caso. Mostra-se que ambas as abordagens podem garantir a contração da função custo, levando, assim, à convergência do sistema de malha fechada tanto para casos de busca de referência quanto para casos de distúrbios não medidos.
\end{abstract}

Keywords: Model Predictive Control; Integrating systems; Robust stability; Electromechanical systems

Palavras-chaves: Controle preditivo; Sistemas integradores; Estabilidade robusta; Sistemas Eletromecânicos

\section{INTRODUÇÃO}

Os controladores preditivos (MPC), que consideram as incertezas associadas aos modelos na formulação de suas leis de controle, são ditos MPC robustos. A garantia da estabilidade robusta de controladores MPC ainda é uma das maiores dificuldades encontradas por engenheiros de controle tanto na academia quanto em casos práticos (Qin and Badgwell, 2003). Entretanto, grandes avanços foram realizados nos últimos anos com intuito de aperfeiçoar as estratégias de controle preditivo com garantia de estabilidade robusta.

Pode-se citar três principais categorias de MPC robusto na literatura (Martins and Odloak, 2016): i) contração de funções custo, ii) contração de estados associados ao modo

\footnotetext{
* Os autores agradecem a FAPESB (Fundação de Amparo a Pesquisa do Estado da Bahia - Programa de Mestrado Sanduíche Processo $n^{\circ}$ 1915/2018) e ao MCTIC (Ministério da Ciência, Tecnologia, Inovações e Comunicações, $n^{\circ} 11137$ - PROPESQ).
}

dual e iii) Minimização do custo para o pior caso. No que se refere à formulação a partir da contração da função custo, (Badgwell, 1997) se destaca como referência primordial. O autor propõe um controlador a partir da extensão de controladores preditivos com horizontes de predição infinito (IHMPC) por incluir restrições que impedem o crescimento da função custo. Este controlador proposto considera incertezas de múltiplos modelos e é aplicável somente em processos estáveis e em casos reguladores.

Com o intuito de aprimorar a abordagem de Badgwell (1997), Odloak (2004) apresenta um IHMPC para casos de controle por servomecanismo e com perturbações não medidas. A proposta desse novo controlador se baseia na representação de modelos em espaço de estados na formulação incremental das entradas do sistema, proposto por (Rodrigues and Odloak, 2003), eliminando o erro de regime estacionário em apenas uma camada. Ademais, este controlador utiliza variáveis de folga para as restrições terminais rígidas do otimizador, permitindo ampliar o domínio de atração e garantir a factibilidade da solução do 
problema, preservando ainda as propriedades estabilizantes da lei de controle.

A partir de Odloak (2004), diversos esforços se destacam no aprimoramento da técnica proposta, como a ampliação para casos de sistemas com polos integradores (González et al., 2007) e para sistemas instáveis integrados ao controle por faixa e busca de alvos econômicos (Martins and Odloak, 2016). Ambos os casos apresentam as propriedades estabilizantes nas quais as funções custo são formuladas de forma a serem limitadas e decrescentes, tal qual uma função de Lyapunov, além de considerarem restrições terminais suavizadas para cancelar os efeitos dos modos integradores e instáveis do sistema.

Ocorre que, em uma análise abrangente, é comum encontrar em sistemas eletromecânicos dinâmicas que apresentam polos integradores repetidos. Neste aspecto, Costa (2017) propõe uma formulação de modelo baseado na resposta ao degrau para sistemas com múltiplos polos integradores e sintetiza uma estratégia de controle preditivo associada. Este controlador segue os conceitos propostos por Carrapiço and Odloak (2005) para garantia da estabilidade nominal em malha fechada, sendo formulado em uma e também em duas camadas (Costa et al., 2018).

Entretanto, o desempenho do controlador nominalmente estável proposto por Costa (2017) é comprometido quando implementado em alguns casos mismatch principalmente quando o sistema opera em condições diferentes daquelas utilizadas para obter o modelo linear interno do controlador.

Sendo assim, como forma de ampliar os resultados alcançados por Costa et al. (2018), este trabalho apresenta a formulação de um controlador preditivo de horizonte infinito com a garantia de estabilidade robusta (RIHMPC) para sistemas com múltiplos polos integradores que apresentam incertezas multi-plantas. O trabalho considera dois casos de formulação da lei de controle: i) a utilização de uma matriz de ponderação de ordem de grandeza elevada para minimização das variáveis de folga associadas aos polos integradores repetidos e ii) a contração das variáveis de folgas a partir de uma restrição não-linear. Os resultados são demonstrados utilizando um sistema quadricóptero de eixo fixo, Hover, quando há inclusão de incertezas na formulação do modelo.

\section{MODELO PARA POLOS INTEGRADORES REPETIDOS}

Nesta seção, será apresentado o modelo em espaço de estados para múltiplos polos integradores tal como proposto por Costa (2017). A formulação deste controlador é baseada na modelagem específica do sistema a partir da parametrização da curva de resposta ao degrau.

O modelo do sistema pode ser representado sob a seguinte forma de espaço de estados:

$$
\left[\begin{array}{c}
\mathbf{x}^{s}(k+1) \\
\mathbf{x}^{i}(k+1)
\end{array}\right]=\underbrace{\left[\begin{array}{cc}
\mathbf{I}_{n y} & \boldsymbol{\tau} \\
\mathbf{0} & \boldsymbol{F}
\end{array}\right]}_{\mathbf{A}}\left[\begin{array}{c}
\mathbf{x}^{s}(k) \\
\mathbf{x}^{i}(k)
\end{array}\right]+\underbrace{\left[\begin{array}{l}
\mathbf{B}^{s} \\
\mathbf{B}^{i}
\end{array}\right]}_{\mathbf{B}} \boldsymbol{\Delta u ( k ) ,}
$$

$$
\mathbf{y}(k)=\underbrace{\left[\begin{array}{ll}
\mathbf{I}_{n y} & \mathbf{0}
\end{array}\right]}_{\mathbf{C}}\left[\begin{array}{l}
\mathbf{x}^{s}(k) \\
\mathbf{x}^{i}(k)
\end{array}\right],
$$

Os estados $\mathbf{x}^{s}$ e $\mathbf{x}^{i}$ são, respectivamente, os estados integradores artificiais, dada a formulação incremental do modelo, e os estados integradores naturais do sistema e que podem se repetirem $n i$ vezes. Para este modelo, ny representa a quantidade de saídas do sistema e $n u$ a quantidade de entradas. $n d=n u \cdot n i \cdot n y . \mathbf{0}$ é uma matriz de zeros de dimensões associada a $n u$ e $n i$.

As matrizes auxiliares ao modelo $\boldsymbol{\tau}, \mathbf{F}, \mathbf{B}^{s}$ e $\mathbf{B}^{i}$ tem dimensões $(n y \times n i),(n i \times n i),(n y \times n u)$ e $(n d \times n u)$, respectivamente, e suas definições podem ser encontradas no trabalho referenciado (Costa, 2017). De forma sucinta, as matrizes $\boldsymbol{\tau}$ e $\mathbf{F}$ são associadas somente aos estados e aos tempos de amostragem do modelo. Já as $\mathbf{B}^{s}$ e $\mathbf{B}^{i}$ são associadas aos parâmetros físicos do sistema os quais relacionam entradas e saídas.

Em Costa et al. (2018), os autores propõem a utilização do modelo apresentado para formulação de um controlador preditivo com estabilidade nominal garantida. Como forma de estender os resultados anteriores, será apresentada a formulação de um controlador preditivo que considere as incertezas de modelo e garanta a estabilidade robusta do sistema em malha-fechada.

\section{FORMULAÇÃO DO RIHMPC}

Para formular o controlador robustamente estável serão consideradas as incertezas de modelo do tipo multi-planta, tal qual apresentado por (Badgwell, 1997), de modo que o conjunto de possíveis modelos de planta é definido como $\boldsymbol{\Omega}=\left(\boldsymbol{\Theta}_{1}, \ldots, \boldsymbol{\Theta}_{L}\right)$, o que pode representar o processo em diversos pontos de operação.

Dado o modelo apresentado em Eq. (2), tem-se que as únicas matrizes que assumem incertezas são as submatrizes $\mathbf{B}^{s}$ e $\mathbf{B}^{i}$, já que as demais estão associadas somente aos estados e a amostragem do sistema. Logo, o conjunto individual de modelos pode ser escrito como $\boldsymbol{\Theta}_{n}=\left\{\mathbf{B}_{n}^{s}, \mathbf{B}_{n}^{i}\right\}$, sendo $n=1, \ldots, L$ a quantidade de possíveis modelos. Adota-se para o caso do modelo que representa a condição mais provável do sistema o conjunto $\boldsymbol{\Theta}_{N}$ e o modelo mais próximo da planta como o conjunto $\boldsymbol{\Theta}_{T}$.

Deste modo, o controlador robusto de horizonte infinito é formulado visando resolver o seguinte problema de otimização:

\section{Problema P1:}

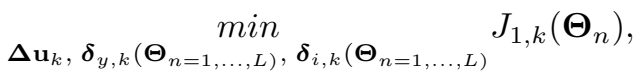

em que: 


$$
\begin{array}{r}
J_{1, k}\left(\boldsymbol{\Theta}_{n}\right)=\sum_{j=1}^{\infty} \| \mathbf{y}_{n}(k+j \mid k)-\mathbf{y}_{s p}-\boldsymbol{\delta}_{y, k}\left(\boldsymbol{\Theta}_{n}\right) \\
-\boldsymbol{\tau} \boldsymbol{\Gamma}_{(j-m)} \boldsymbol{\delta}_{i, k}\left(\boldsymbol{\Theta}_{n}\right)\left\|_{\mathbf{Q}_{y}}^{2}+\sum_{j=0}^{m-1}\right\| \boldsymbol{\Delta} \mathbf{u}(k+j \mid k) \|_{\mathbf{R}}^{2} \\
+\left\|\boldsymbol{\delta}_{y, k}\left(\boldsymbol{\Theta}_{n}\right)\right\|_{\mathbf{S}_{y}}^{2}+\left\|\boldsymbol{\delta}_{i, k}\left(\boldsymbol{\Theta}_{n}\right)\right\|_{\mathbf{S}_{i}}^{2}, \\
n=1, \ldots, L,
\end{array}
$$

sujeito à :

$$
\begin{gathered}
U=\left\{\begin{array}{c}
\Delta \mathbf{u}_{\min } \leq \boldsymbol{\Delta} \mathbf{u}(k+j \mid k) \leq \boldsymbol{\Delta} \mathbf{u}_{\max }, \\
\boldsymbol{\Delta} \mathbf{u}(k+j \mid k)=0, j>m, \\
\mathbf{u}_{\min } \leq \mathbf{u}(k+j \mid k) \leq \mathbf{u}_{\max },
\end{array}\right. \\
\mathbf{x}^{s}(k+m \mid k)-\mathbf{y}_{s p}-\boldsymbol{\delta}_{y, k}\left(\boldsymbol{\Theta}_{n=1, \ldots, L}\right)=0 \\
\mathbf{x}^{i}(k+m \mid k)-\boldsymbol{\delta}_{i, k}\left(\boldsymbol{\Theta}_{n=1, \ldots, L}\right)=0 \\
J_{1, k}\left(\boldsymbol{\Theta}_{n}\right) \leq \widetilde{J}_{1, k}\left(\boldsymbol{\Theta}_{n=1, \ldots, L}\right)
\end{gathered}
$$

onde os custos associados à $\widetilde{J}_{1, k}\left(\boldsymbol{\Theta}_{n=1, \ldots, L}\right)$ são calculados com as ações de controle ótimas herdadas do Problema $\mathbf{P 1}$ no instante anterior $k-1$

$$
\begin{gathered}
\boldsymbol{\Delta} \widetilde{\mathbf{u}}_{k}=\left[\boldsymbol{\Delta} \mathbf{u}^{*}(k \mid k-1)^{\top} \ldots\right. \\
\left.\Delta \mathbf{u}^{*}(k+m-2 \mid k-1)^{\top} \mathbf{0}^{\top}\right]^{\top},
\end{gathered}
$$

As variáveis de folga $\widetilde{\boldsymbol{\delta}}_{y, k}$ e $\widetilde{\boldsymbol{\delta}}_{i, k}$ são calculadas de modo que seja possível satisfazer as restrições de igualdade das Eq. (5) e (6). Os estados atuais da planta, $\mathbf{x}(k)$, são realimentados para todo o conjunto $\Omega$, o que garante que a restrição da Eq. (7) tenha os seus valores sempre atualizados para todos os modelos de planta.

Observação 1: A solução do Problema P1 exige uma programação não-linear (NLP - Non-Linear Programming) devido à restrição da Eq. (7). Entretanto, o problema proposto é um problema convexo dado o fato de que a restrição associadas à contração das funções-custo é quadrática. Ou seja, a restrição não-linear é convexa o que faz com que a solução sempre convirja a um ótimo global.

A garantia de estabilidade robusta é sumarizada pelo seguinte Teorema.

Teorema 1. Dado um sistema com múltiplos polos integradores cuja representação da planta é tida como incertezas multi-plantas assumindo quaisquer dos possíveis modelos contidos em $\Omega$. Então, se num dado momento $k$ o vetor das variáveis de folga integradoras repetidas for zero, este irá permanecer zero para todos os futuros instantes de amostragem $k+j$. Ademais, se o processo é controlável no estado estacionário desejado, a solução do Problema P1 apresentará ações de controle que conduzirá o modelo real da planta ao ponto de equilíbrio desejado de forma estável e assintoticamente convergente.

Prova. Supondo que no instante de amostragem $k$ o Problema P1 apresenta uma solução ótima:

$$
\boldsymbol{\Delta} \mathbf{u}_{k}^{*}, \boldsymbol{\delta}_{y, k}^{*}\left(\boldsymbol{\Theta}_{n=1, \ldots, L}\right), \boldsymbol{\delta}_{i, k}^{*}\left(\boldsymbol{\Theta}_{n=1, \ldots, L}\right)=0
$$

sendo,

$$
\begin{array}{r}
\Delta \mathbf{u}_{k}^{*}=\left[\boldsymbol{\Delta} \mathbf{u}^{*}(k \mid k)^{\top} \boldsymbol{\Delta} \mathbf{u}^{*}(k+1 \mid k)^{\top} \ldots\right. \\
\left.\boldsymbol{\Delta} \mathbf{u}^{*}(k+m-1 \mid k)^{\top}\right]^{\top},
\end{array}
$$

O valor da função custo para o modelo real da planta $(n=T)$ será:

$$
\begin{array}{r}
J_{1, k}^{*}\left(\boldsymbol{\Theta}_{T}\right)=\sum_{j=1}^{\infty} \| \mathbf{y}_{T}(k+j \mid k)-\mathbf{y}_{s p}-\boldsymbol{\delta}_{y, k}^{*}\left(\boldsymbol{\Theta}_{T}\right) \\
-\boldsymbol{\tau} \boldsymbol{\Gamma}_{(j-m)} \boldsymbol{\delta}_{i, k}^{*}\left(\boldsymbol{\Theta}_{T}\right)\left\|_{\mathbf{Q}_{y}}^{2}+\sum_{j=0}^{m-1}\right\| \boldsymbol{\Delta}^{*}(k+j \mid k) \|_{\mathbf{R}}^{2} \\
+\left\|\boldsymbol{\delta}_{y, k}^{*}\left(\boldsymbol{\Theta}_{T}\right)\right\|_{\mathbf{S}_{y}}^{2},
\end{array}
$$

Em sequência, para provar a estabilidade do controlador proposto, demonstra-se primeiramente a viabilidade recursiva da solução. Para isso, considera-se que no próximo instante de amostragem $k+1$, assume-se que a solução obtida será aquela solução herdada do instante anterior $k$, sendo:

$$
\begin{array}{r}
\Delta \widetilde{\mathbf{u}}_{k+1}=\left[\boldsymbol{\Delta} \mathbf{u}^{*}(k+1 \mid k)^{\top} \boldsymbol{\Delta} \mathbf{u}^{*}(k+2 \mid k)^{\top}\right. \\
\left.\ldots \boldsymbol{\Delta} \mathbf{u}^{*}(k+m-1 \mid k)^{\top} \mathbf{0}^{\top}\right]^{\top},
\end{array}
$$

cujas variáveis de folga $\widetilde{\boldsymbol{\delta}}_{y, k+1}\left(\boldsymbol{\Theta}_{n=1, \ldots, L}\right)$ e $\widetilde{\boldsymbol{\delta}}_{i, k+1}\left(\boldsymbol{\Theta}_{n=1, \ldots, L}\right)$ serão tais que satisfaçam as equações

$$
\begin{array}{r}
\mathbf{x}^{s}(k+m \mid k+1)-\mathbf{y}_{s p}-\widetilde{\boldsymbol{\delta}}_{y, k+1}\left(\boldsymbol{\Theta}_{n=1, \ldots, L}\right)=0 \\
\mathbf{x}^{i}(k+m \mid k+1)-\widetilde{\boldsymbol{\delta}}_{i, k}\left(\boldsymbol{\Theta}_{n=1, \ldots, L}\right)=0
\end{array}
$$

Observa-se que, para o modelo de planta real $\Theta_{T}$, a solução herdada do instante $k$ será uma solução factível no instante $k+1$. Além disso, como as ações de controle são as mesmas para todos os modelos e os estados realimentados são os próprios estados da planta real, pode-se deduzir que a predição dos estados no instante $k+1$ é igual a predição em $k$, isto é: $\mathbf{x}_{T}(k+j \mid k+1)=\mathbf{x}_{T}(k+j \mid k)$. Assim, conclui-se que as variáveis de folga herdadas no instante $k+1$ serão iguais àquelas no instante $k: \widetilde{\boldsymbol{\delta}}_{y, k+1}\left(\boldsymbol{\Theta}_{T}\right)=\boldsymbol{\delta}_{y, k}^{*}\left(\boldsymbol{\Theta}_{T}\right)$, $\widetilde{\boldsymbol{\delta}}_{i, k+1}\left(\boldsymbol{\Theta}_{T}\right)=\boldsymbol{\delta}_{i, k}^{*}\left(\boldsymbol{\Theta}_{T}\right)=\mathbf{0}$.

Destarte, para provar a convergência da função objetivo, deve-se avaliar o valor da função custo para o modelo de planta real dadas as soluções propostas anteriormente:

$$
\begin{array}{r}
\widetilde{J}_{1, k+1}\left(\boldsymbol{\Theta}_{T}\right)=\sum_{j=1}^{\infty} \| \mathbf{y}_{T}(k+j+1 \mid k)-\mathbf{y}_{s p}-\boldsymbol{\delta}_{y, k}^{*}\left(\boldsymbol{\Theta}_{T}\right) \\
-\boldsymbol{\tau} \boldsymbol{\Gamma}_{(j-m)} \boldsymbol{\delta}_{i, k}^{*}\left(\boldsymbol{\Theta}_{T}\right)\left\|_{\mathbf{Q}_{y}}^{2}+\sum_{j=0}^{m-1}\right\| \boldsymbol{\Delta} \mathbf{u}^{*}(k+j+1 \mid k) \|_{\mathbf{R}}^{2} \\
+\left\|\boldsymbol{\delta}_{y, k}^{*}\left(\boldsymbol{\Theta}_{T}\right)\right\|_{\mathbf{S}_{y}}^{2}
\end{array}
$$

A diferença entre Eq. 10 e Eq. 12 resulta somente nos valores das variáveis no instante $k$ dado como: 


$$
\begin{aligned}
J_{1, k}^{*}\left(\boldsymbol{\Theta}_{T}\right)-\widetilde{J}_{1, k+1}\left(\boldsymbol{\Theta}_{T}\right)= & \left\|\mathbf{y}(k+1 \mid k)-\mathbf{y}_{s p}-\boldsymbol{\delta}_{y, k}^{*}\left(\boldsymbol{\Theta}_{T}\right)\right\|_{\mathbf{Q}_{y}}^{2} \\
& +\left\|\boldsymbol{\Delta} \mathbf{u}^{*}(k \mid k)\right\|_{\mathbf{R}}^{2}
\end{aligned}
$$

Observa-se que os termos à direita da Eq. (13) sempre serão positivos, já que as matrizes $\mathbf{Q}_{y}$ e $\mathbf{R}$ são positivas definidas. Através da restrição descrita na Eq. (7), pode-se observar que $J_{1, k+1}^{*}\left(\boldsymbol{\Theta}_{n}\right) \leq \widetilde{J}_{1, k+1}\left(\boldsymbol{\Theta}_{n}\right)$. Logo:

$$
J_{1, k+1}^{*}\left(\boldsymbol{\Theta}_{T}\right) \leq J_{1, k}^{*}\left(\boldsymbol{\Theta}_{T}\right)
$$

Sendo assim, conclui-se que os instantes subsequentes a $k$ sempre terão soluções cujo valor da função custo será menor que o instante anterior, provando a convergência do controlador proposto tal qual uma função de Lyapunov.

A convergência monotônica da função custo será sempre para o modelo da planta real, podendo os outros modelos não obedecerem à esta condição. Ademais, a prova dessa convergência exige que as variáveis de folga $\boldsymbol{\delta}_{i, k}^{*}\left(\boldsymbol{\Theta}_{T}\right)$ sejam zeradas, o que neste caso, dada a Eq (6), exige que os estados integradores repetidos do processo sejam zero. Logo, satisfazendo esta condição, haverá a convergência da função custo nos instantes de amostragem subsequentes. Caso contrário, as variáveis de folga do otimizador não serão zeradas, o que ocasionará um erro de estado estacionário e um valor de $J_{1, k}>0$.

A garantia da convergência das variáveis integradoras repetidas a zero é dada pelo uso de pesos altos na matriz de ponderação $S_{i}$. De forma a demonstrar que esta condição é suficiente para alcançar a estabilidade de sistemas com múltiplos polos integradores, é proposto um novo problema de otimização. Neste caso, leva-se em consideração a contração das variáveis de folga dos estados integradores repetidos, adicionando explicitamente uma condição que force a convergência a zero dos estados integradores. Destarte, o novo problema de otimização é formulado da seguinte forma:

\section{Problema P2:}

$$
\min _{\boldsymbol{\Delta} \mathbf{u}_{k}, \boldsymbol{\delta}_{y, k}\left(\boldsymbol{\Theta}_{n}=1, \ldots, L\right), \boldsymbol{\delta}_{i, k}\left(\boldsymbol{\Theta}_{n=1, \ldots, L)}\right.} J_{2, k}\left(\boldsymbol{\Theta}_{n}\right),
$$

em que:

$$
\begin{array}{r}
J_{2, k}\left(\boldsymbol{\Theta}_{n}\right)=\sum_{j=1}^{\infty} \| \mathbf{y}_{n}(k+j \mid k)-\mathbf{y}_{s p}-\boldsymbol{\delta}_{y, k}\left(\boldsymbol{\Theta}_{n}\right) \\
-\boldsymbol{\tau} \boldsymbol{\Gamma}_{(j-m)} \boldsymbol{\delta}_{i, k}\left(\boldsymbol{\Theta}_{n}\right)\left\|_{\mathbf{Q}_{y}}^{2}+\sum_{j=0}^{m-1}\right\| \boldsymbol{\Delta} \mathbf{u}(k+j \mid k) \|_{\mathbf{R}}^{2} \\
\left\|\boldsymbol{\delta}_{y, k}\left(\boldsymbol{\Theta}_{n}\right)\right\|_{\mathbf{S}_{y}}^{2}+\left\|\boldsymbol{\delta}_{i, k}\left(\boldsymbol{\Theta}_{n}\right)\right\|_{\mathbf{S}_{i}}^{2} \\
n=1, \ldots, L
\end{array}
$$

sujeito à (4), (5), (6) e (7) e:

$$
\left\|\boldsymbol{\delta}_{i, k}\left(\boldsymbol{\Theta}_{n}\right)\right\|_{\mathbf{S}_{i}}^{2} \leq\left\|\widetilde{\boldsymbol{\delta}}_{i, k}\left(\boldsymbol{\Theta}_{n=1 \ldots L}\right)\right\|_{\mathbf{S}_{i}}^{2},
$$

Observação 2: Supondo que em um dado instante $\bar{k}$ as variáveis de folga integradoras são zeradas. Deste modo, o Problema P2 resume-se no mesmo Problema P1, cujo teorema e prova já foram apresentados anteriormente.

Observação 3: Devido à inclusão de mais uma restrição não-linear para contração das variáveis de folga dos polos integradores repetidos, mesmo sendo um problema de otimização convexo, aumenta-se a complexidade do programa de otimização, o que na prática se traduz em $L \times n i$ variáveis para solução do problema. Consequentemente, o Problema P2 apresenta maior complexidade que o Problema P1.

\section{SIMULAÇÃO DO RIHMPC PARA MÚLTIPLOS POLOS INTEGRADORES}

O propósito desta seção é apresentar os resultados de simulação e avaliar os desempenhos dos controladores propostos, utilizando, para isso, o mesmo sistema trabalhado por Costa et al. (2018) quando inserido incertezas de modelagem. O modelo para testes representa um quadricóptero de eixo fixo, comumente chamado de Hover, o qual consiste de quatro motores propulsores que alteram os ângulos de inclinação do eixo (arfagem, rolagem e guinada). O objetivo de controle são os ângulos de inclinação do eixo do conjunto, manipulando assim as tensões dos quatro propulsores. Portanto, propõe-se a simulação de dois controladores: Controlador $\mathbf{1}$ - conforme proposto no Problema 1 e Controlador 2- conforme proposto no Problema 2.

Neste cenário, considera-se que o modelo da função de transferência que mais se aproxima do sistema real será $\boldsymbol{\Theta}_{1}(s)$, definido a partir da modelagem fenomenológica e substituído os valores das constantes conforme manual do fabricante (Quanser, 2011). Já os modelos $\boldsymbol{\Theta}_{2}(s)$ e $\boldsymbol{\Theta}_{3}(s)$ são modelos cujas incertezas de $+25 \%$ e $-25 \%$, respectivamente, foram consideradas na constante de torque dos motores propulsores.

$$
\begin{gathered}
\Theta_{1}(s)=\left[\begin{array}{cccc}
\frac{-0.03273}{s^{2}} & \frac{-0.03273}{s^{2}} & \frac{0.03273}{s^{2}} & \frac{0.03273}{s^{2}} \\
\frac{0.4240}{s^{2}} & \frac{-0.42}{s^{2}} & 0 & 0 \\
0 & 0 & \frac{0.4240}{s^{2}} & \frac{-0.4240}{s^{2}}
\end{array}\right] . \\
\Theta_{2}(s)=\left[\begin{array}{cccc}
\frac{-0.0409}{s^{2}} & \frac{-0.0409}{s^{2}} & \frac{0.00 .0409}{s^{2}} & \frac{0.0 .0409}{s^{2}} \\
\frac{0.5300}{s^{2}} & \frac{-0.5300}{s^{2}} & 0 & 0 \\
0 & 0 & \frac{0.5300}{s^{2}} & \frac{-0.5300}{s^{2}}
\end{array}\right] . \\
\Theta_{3}(s)=\left[\begin{array}{cccc}
\frac{-0.0262}{2} & \frac{-0.0262}{s^{2}} & \frac{0.0262}{s^{2}} & \frac{0.0262}{s^{2}} \\
\frac{0.3392}{s^{2}} & \frac{-0.3392}{s^{2}} & 0 & 0 \\
0 & 0 & \frac{0.3392}{s^{2}} & \frac{-0.3392}{s^{2}}
\end{array}\right] .
\end{gathered}
$$

Foi definido o tempo de amostragem para parametrização do modelo $\Delta t=0.1 \mathrm{~s}$. O estado estacionário inicial do sistema ocorre para as quatro entradas $\mathbf{u}_{s s}=[2,2,2,2]^{\top} \mathrm{V}$ $\mathrm{e}$ as três saídas $\mathbf{y}_{s s}=\left[\begin{array}{lll}0, & 0, & 0\end{array}\right]^{\top} \mathrm{rad}$. Para fins e comparação, a sintonia para os dois controladores possuem os seguintes parâmetros: $m=3, \mathbf{Q}_{y}=10^{-3} \operatorname{diag}(n y)$, $\mathbf{R}=10^{-3} \operatorname{diag}(n u), \mathbf{S}_{y}=10^{6} \operatorname{diag}(n y), \mathbf{S}_{i}=10^{6} \operatorname{diag}(n i)$ $, \mathbf{y}_{\max }=[\pi / 2, \pi / 2, \pi / 2, \pi / 2]^{\top}[\mathrm{rad}], \mathbf{y}_{\min }=-\mathbf{y}_{\max }$, $\mathbf{u}_{\max }=24 \cdot[1,1,1,1]^{\top}[\mathrm{V}], \mathbf{u}_{\min }=[0,0,0,0]^{\top}[\mathrm{V}]$ e $\boldsymbol{\Delta} \mathbf{u}=$ $6 .[1,1,1,1][\mathrm{V}]$. 
Para o caso de busca de referência, simula-se uma mu-

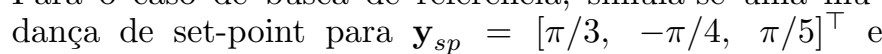
$\mathbf{y}_{s p}=\left[\begin{array}{lll}\pi / 5, & \pi / 4, & 0\end{array}\right]^{\top}$ nos instantes $t=1$ s e $t=13$ $\mathrm{s}$, respectivamente. Um distúrbio não medido, do tipo impulso, é simulado nas entradas em $t=10 \mathrm{~s}$, com amplitude $d=\left[\begin{array}{lll}2.5,-7.5, & 6.0,-6.5\end{array}\right]^{\top}$ V. Para esta simulação, é definido o modelo $\boldsymbol{\Theta}_{1}$ como o modelo real da planta e $\boldsymbol{\Theta}_{2}$ como o modelo nominal dos controladores.

A figura 1 apresenta as saídas dos dois controladores. Observa-se, graficamente, que os controladores propostos apresentam desempenho equivalente, havendo pouca diferença quando há distúrbio no processo. Neste caso, o Controlador 1 apresenta uma resposta um pouco mais lenta do que o Controlador 2. Já a figura 2 mostra as entradas do sistema para ambos os controladores. Da mesma forma, é imperceptível as diferenças entre as variáveis de entrada dos controladores propostos.
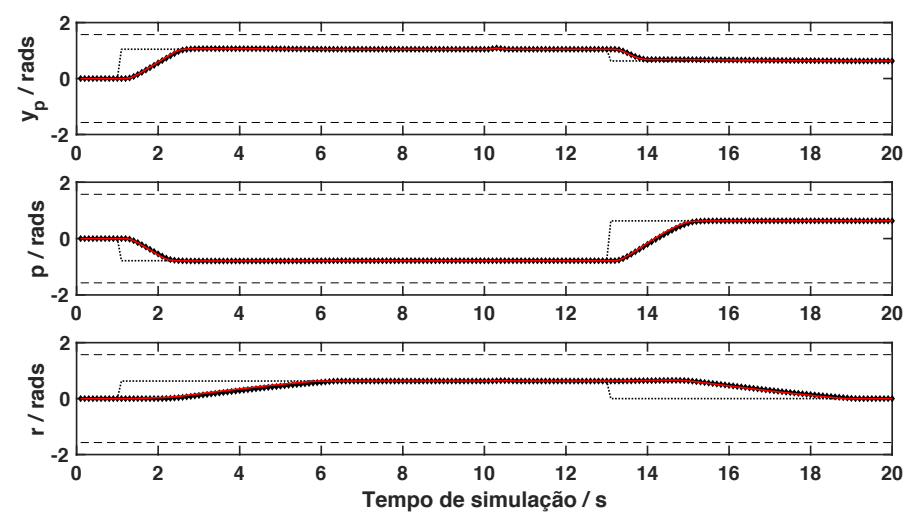

Figura 1. Saída do sistema para mudança de variável e absorção de distúrbio. (- - - Limites superior e inferior; ... Referência; - Controlador 1; - - - Controlador 2).

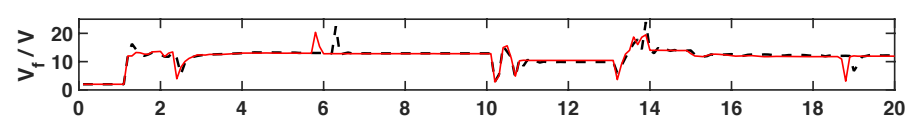

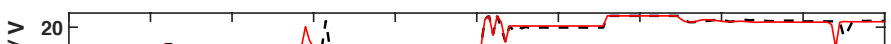

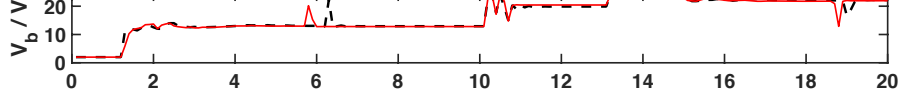
2
$>$

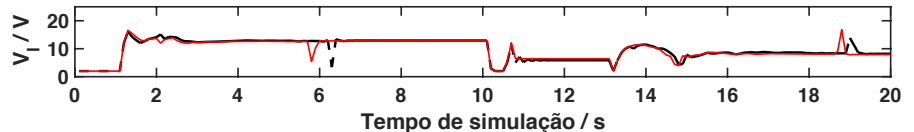

Figura 2. Entradas do sistema para mudança de variável e absorção de distúrbio. (- Controlador 1; - - Controlador 2).

A figura 3 mostra o valor das variáveis de folga relacionadas aos polos integradores e associada ao modelo referente à planta $\left(\Theta_{T}\right)$ para ambos os controladores. Para facilitar o entendimento, essas variáveis foram somadas ao seus quadrados em cada instante de amostragem, obtendo o valor total para todos os estados integradores em cada instante de simulação $\left(\sum_{j=1}^{n i} \delta_{i n, j}^{2}, j=1 \ldots t_{\text {final }}\right)$. Ambos os controladores minimizam a utilização dessas variáveis, levando-as a zero. Entretanto, é possível perceber que, para o Controlador 2, os valores das variáveis de folga são pouco menores do que aquelas do Controlador 1. Este fato esta relacionado com a contração das variáveis de folga dado que o Controlador 2 considera esta condição tal como proposto na Eq. (16) do Problema P2, diferentemente do Controlador 1, que somente considera a contração da função custo.

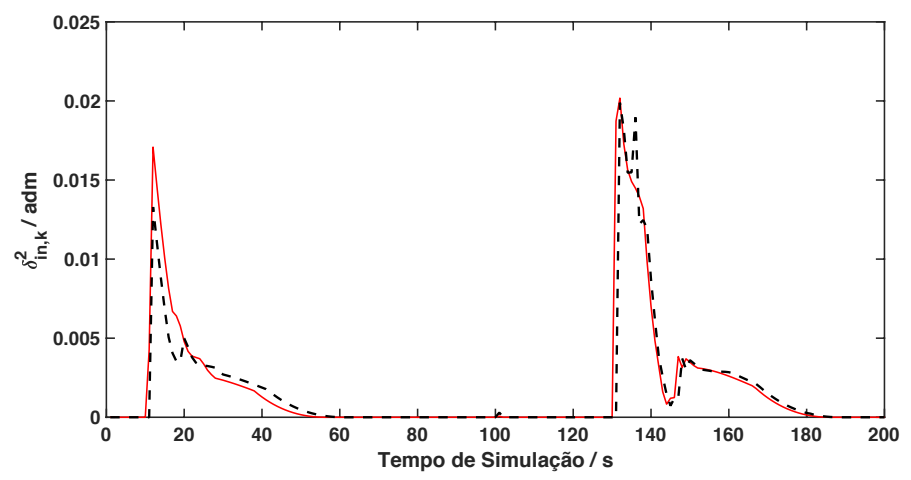

Figura 3. Variáveis de folga $\boldsymbol{\delta}_{i, k}$ para cada saída. (Controlador 1; - - Controlador 2).

Conforme mostrado na figura 4, a função custo associada ao modelo da planta $\left(\Theta_{T}\right)$ é assintoticamente decrescente para os dois controladores, obedecendo a garantia de estabilidade tanto do Controlador 1 quanto do Controlador 2 .
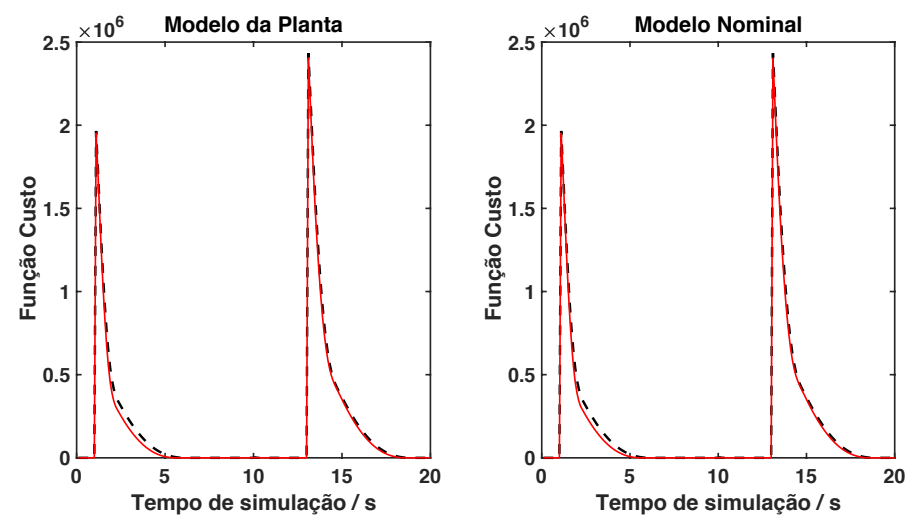

Figura 4. Valor da Função Objetivo. (- Controlador 1; - - Controlador 2).

A tabela 1 mostra o desempenho dos otimizadores e o índice de desempenho dos controladores propostos. Os dois controladores utilizaram o algoritmo de otimização programação quadrática sequencial (SQP) para solução do problema. Para análise de desempenho, foi considerado o ITAE (Integral do Erro Absoluto multiplicado pelo Tempo) como a soma total das três saídas.

Observa-se que o Controlador 2 apresenta os índices de desempenho do otimizador maiores do que o Controlador 1. Esse resultado já é esperado dado que o Controlador 2 apresenta uma restrição não linear a mais, aumentando a complexidade de solução do otimizador e assim o tempo de simulação. Portanto, a adição da restrição de contração das variáveis de folga associadas aos estados integradores do sistema propostas no Problema P2, pode ser negligenciada por dois fatores: (i) a minimização das variáveis 
Tabela 1. Tabela de Comparação de desempenho dos controldores

\begin{tabular}{|c|c|c|}
\hline $\begin{array}{c}\text { Índice de } \\
\text { desempenho }\end{array}$ & Controlador 1 & Controlador 2 \\
\hline ITAE $\left[\right.$ rads $\left.^{2}\right]$ & 6956.80 & 7372.00 \\
\hline $\begin{array}{c}\text { Máximo } \\
\text { de interações / } \\
\text { amostragem }\end{array}$ & 115 & 85 \\
\hline $\begin{array}{c}\text { Total } \\
\text { de interações }\end{array}$ & 3725 & 4964 \\
\hline $\begin{array}{c}\text { Tempo } \\
\text { de simulação }\end{array}$ & $104.76 \mathrm{~s}$ & $139.03 \mathrm{~s}$ \\
\hline
\end{tabular}

de folgas podem ser garantidas a partir da utilização de pesos na ordem de grandeza superior a $10^{4}$ na matriz de ponderação $S_{i}$, (ii) o desempenho de controle com a contração das variáveis de folga é muito semelhante ao desempenho sem esta restrição. Ademais, em processos reais, a quantidade de variáveis utilizadas tende a ser maior, o que faz com que o custo computacional aumente exponencialmente, o que corrobora para a utilização do Controlador 1 em cenários práticos.

\section{CONCLUSÃO}

Este trabalho apresenta um arcabouço de controle preditivo com estabilidade robusta garantida para sistemas com polos integradores repetidos. A garantia de estabilidade depende da convergência dos estados associados aos polos integradores a zero. Nesse sentido, duas formulações foram propostas ao esquema de controle. Ambas consideram a contração da função custo como restrição não-linear do otimizador. Entretanto, a primeira formulação utiliza a matriz de ponderação das variáveis de folga associadas aos polos integradores $\left(S_{i}\right)$ com ordem de grandeza muito superior das demais matrizes enquanto a segunda formulação adiciona uma restrição para contração das variáveis de folga integradoras.

Com base nos resultados obtidos por simulação, observase que os dois controladores satisfazem a condição de estabilidade robusta, tanto para casos de busca de referência quanto para rejeição de distúrbios. A formulação do problema de otimização com restrição de contração das variáveis de folga apresenta desempenho semelhante ao problema sem esta restrição. Deste modo, face ao maior custo computacional devido à adição de mais uma restrição não linear, mesmo que convexa, pode-se negligenciar esta condição e utilizar somente a correta ponderação na matriz de pesos das variáveis de folga integradoras.

Finalmente, o controlador proposto se mostra atrativo e plausível de implementação real e vislumbra-se, em trabalhos futuros, a sua aplicação em casos práticos, bem como a comparação de desempenho com outros controladores com aspectos de controle robusto para sistemas de múltiplos polos integradores.

\section{REFERÊNCIAS}

Badgwell, T.A. (1997). Robust model predictive control of stable linear systems. Int. J. Control, 68, 797-818.

Carrapiço, O.L. and Odloak, D. (2005). A stable model predictive control for integrating processes. Computers and Chemical Engineering, 29(5), 1089-1099. doi:10. 1016/j.compchemeng.2004.11.008.
Costa, E.A. (2017). Controle preditivo com estabilidade nominal garantida para sistemas integradores com polos repetidos. Mestrado, Universidade Federal da Bahia.

Costa, E.A., Pataro, I.M.L., de Almeida, F.S.P., and Martins, M.A.F. (2018). Controlador preditivo com estabilidade garantida em duas camadas para sistemas com polos integradores repetidos. In XXII Congresso Brasileiro de Automática, 732-737. UFPB, João Pessoa - PB.

González, A.H., Marchetti, J.L., and Odloak, D. (2007). Extended robust model predictive control of integrating systems. AIChE Journal, 53(7), 1758-1769. doi:10. 1002 /aic.11196.

Martins, M.A.F. and Odloak, D. (2016). A robustly stabilizing model predictive control strategy of stable and unstable processes. Automática, 67, 132 - 143 .

Odloak, D. (2004). Extended robust model predictive control. AIChE Journal, 50(8), 1824-1836. doi:10.1002/ aic. 10175.

Qin, S. and Badgwell, T.A. (2003). A survey of industrial model predictive control technology. Control Engineering Practice, 11(7), 733 - 764. doi:https://doi.org/10. 1016/S0967-0661(02)00186-7.

Quanser (2011). Student workbook, rotary servo base unit experiment for matlab $\AA /$ simulink users.

Rodrigues, M.A. and Odloak, D. (2003). MPC for stable linear systems with model uncertainty. Automatica, 39(4), 569-583. doi:10.1016/S0005-1098(02)00176-0. 